\title{
SPECIFIC ASPECTS OF ACTIVE AND SEMI-ACTIVE SUSPENSIONS
}

\author{
Martin Maloch, Stefan Cornak \\ University of Defence in Brno, Czech Republic \\ martin.maloch@unob.cz, stefan.cornak@unob.cz
}

\begin{abstract}
This paper is concerned with the needs and possibilities of implementing the active and semi-active suspensions for wheeled vehicles of the Army of the Czech Republic (ACR) - for drivetrain configuration $4 \times 4$ to $8 \times 8$. The computer technology is currently on a very high level, the amount of data computed per second is on steep rise and various processes have a capability to be driven almost real-time. The active suspension is nowadays fully implemented in the highest classes of civil cars, therefore, it is time for the implementation into the demands for modernisation of the wheeled vehicles of ACR. The land relief of central Europe is significantly mountainous, thus the demands for the wheeled vehicles, in terms of overcoming obstacles, are very high. After the application of the active suspension, the mobility and driving comfort of the vehicle are greatly enhanced. Unfortunately, at the cost of increased energy consumption. Therefore, the amount of energy available for the drivetrain is significantly lowered. On the other hand, the amount of energy required by the semi-active suspension is remarkably lower. The mobility and driving comfort are better than a passive suspension. Thus, it is recommendable to use scanning of the land relief in front of the vehicle subsequently controlling the chosen elements of the suspension by suitable algorithms. The aim of this study is to create an analysis of accessible information about the applications and control methods of active and semi-active suspensions. Evaluation of the wide range of possibilities was done with regard to the crew of the vehicle. The crew is facing problems from the fatigue and incidence of motion sickness to the worst case scenario, failure of organs.
\end{abstract}

Keywords: active suspension, semi-active suspension, vibrations.

\section{Introduction}

Safety, handling and road stability are guaranteed by appropriate vehicle suspension, consisting of a springing element and a damping element. Separately, these elements are rather useless, the damper has no bearing function and the vehicle with springs only would behave like a boat on the open sea. Initially, the vehicle suspensions were equipped with springing elements (leather belts) only and they were sufficient due to low traveling speeds. The origin of steel springing elements (leaf springs) is connected with the Industrial Revolution and with development of the manufacturing processes. The first vehicle with a hydraulic damper appeared in the early years of the 20th century. The vehicle suspension went through heavy development, but up to date, leaf springs are still utilized, despite certain drawbacks (dimensions, weights). Heavy trucks and trailers, off-road vehicles, dump trucks form a group of vehicles in which leaf springs are still utilized. Coil springs are often used for lighter vehicles, ordinary ones with linear spring characteristics, or modern ones with a progressive one. The achievement of progressive characteristic in coil springs requires significantly higher production costs. In case of heavy military vehicles, torsion bars were put to use. Their stiffness depends on their diameter and length, along with the layout on the vehicle. In modern wheeled vehicles, springing properties of gas, usually enclosed in flexible bellow, are utilized. Used in combination with properties of a hydraulic fluid, the most modern part of the suspension is created - hydro-pneumatic unit. Both media are separated by a flexible membrane with a possibility of regulating the fluid volume under the membrane. Thus, the change of the ride height is attained. Properties of high-pressure gas are used as a springing element, and the flow of hydraulic fluid through a valve (hydraulic resistance) is used as a damping element. Since the movement of the wheel is transferred to the membrane, the hydraulic fluid must be supplied to the system by the hydraulic pump. Working pressures are usually ranging from 10 to $20 \mathrm{MPa}$.

Currently, the best comfort, handling and road stability can be achieved with an independent wheel suspension, usually MacPherson, double wishbone or their variations, along with semi-active or active control elements. In case of a semi-active suspension, the damping characteristic is the control element, whereas with an active suspension, the control element is the springing characteristic. With a passive suspension, for example Fig. 1, the properties of the spring and the damper are set during their assembly, therefore it is not possible to change them during operation. Their characteristics are tuned for a specific weight of the vehicle. However, the weight of the vehicle can vary, from a minimal nominal weight to maximal admissible- fully loaded with cargo and crew. Naturally, a next step in the suspension development was its progressive characteristic, which was progressively changing along 
with the rise of the vehicle weight - cargo, passengers etc. With the rapid improvement of computer aided technologies, control of the suspension characteristics occurred. Control process is done by an operator or by appropriate inputs from ECU of the vehicle.

The role of springing elements is energy accumulation. Without them, impacts and shocks from the wheel would be transferred directly to the frame of the vehicle, thus to the powerpack, crew and cargo. With the successful separation of wheels and chassis, conducted by utilization of the springing element, the mass of the vehicle is divided into sprung and unsprung mass - Fig. 2. Road irregularities and bumps are not transferred directly to the frame, instead the energy created by the wheel travel is stored by the compression of the spring. Afterwards, during expansion, the energy is dissipated by the damper and vice versa.

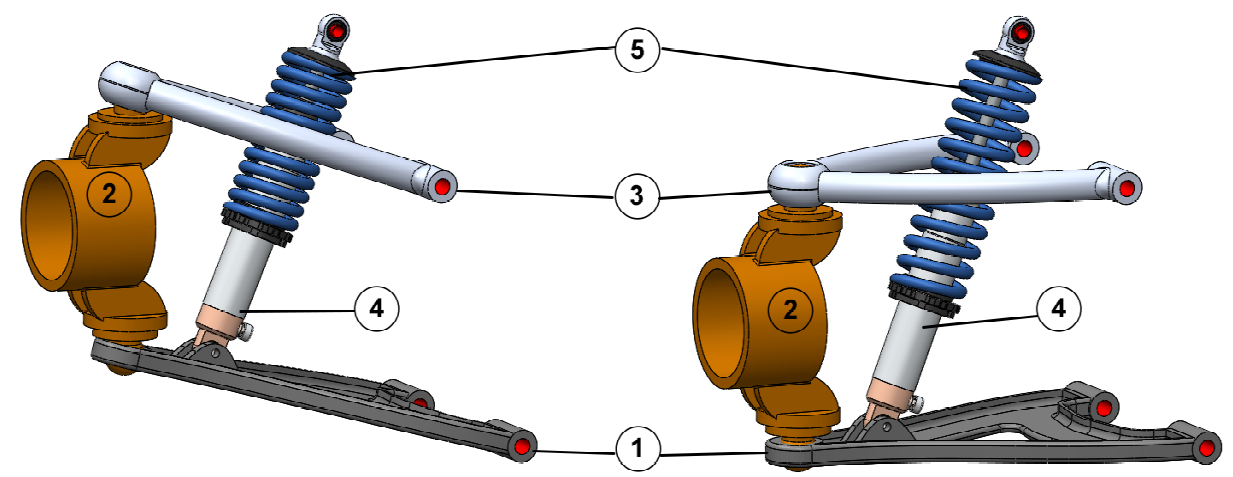

Fig. 1. Illustration of double wishbone suspension unit with coilover layout of spring and shock absorber: 1 - bottom A arm; 2 - spindle; 3 - top A arm; 4 - shock absorber; 5 - spring

Based on the principle of hydraulic resistance, fluid is pushed from the area above the piston through the narrow "valve" to the area under the piston. Thus, the energy is dissipated. With this knowledge, it is obvious that controlling the damping characteristics is a much easier task than the springing characteristics. Hence, the development of suspension continued with a simpler task and the semi-active suspension was proposed. Control of the springing characteristics was achieved later, in various kinds of suspensions and actuators. Since a kind of actuator is needed, the active suspension needs higher energy input from the engine.

\section{Semi-active suspension}

As mentioned before, this type of suspension is the outcome of efforts for an improvement of comfort and road handling with the lowest possible power demand. Since this topic is highly covered, the utilization of the semi-active suspension (SAS) ranges from rail vehicles and trains to civil engineering (huge dampers underneath the base plate of buildings as prevention from earthquakes), and lots of publications are presented every day. Good overall summary is presented in the book [1]. Variety of control algorithms are divided in two main groups - comfort oriented (Skyhook) and roadhandling performance (Groundhook). Few drawbacks are occurring in both types, thus lot of hybrid control algorithms are proposed. Another group of SAS is relying on rheological properties of fluids, magnetorheological (MR) or electrorheological (ER). Viscosity of these fluids is controlled by the amount of magnetic (MR) or electric (ER) field applied. In case of wheeled vehicles, MR dampers are preferred, more studies are oriented on the topic, for example [2], because of low energy requirementsthe battery of the vehicle is sufficient. Lots of scientific publications are aimed for SAS, however, the "engineering" side of the topic should not be forgotten. From the point of view of armoured military wheeled vehicles, for example, armoured personnel carriers (APC), at least one more aspect of suspension has not been mentioned yet: the space requirements. Other demands could be: excellent dependability, maintenance factors- cost, time, crew and proper logistic services with spare parts etc. Last but not the least, the ability of acquisitioning the wheeled military vehicles is divided into two ways. Either, it can be bought anew as a replacement of an obsolete vehicle or the vehicle might be sufficient, but some parts might be outdated, for example, suspension. Thus, it needs to be replaced and modernized. Since a transition from passive to semi-active suspension is done by changing of the damper units, SAS is easily the first choice in case of modernization. Therefore, expensive and demanding design changes are unnecessary. Same rules are applied for hydro-pneumatic units. 


\section{Active suspension}

Controllable springing characteristic is the basic element of the active suspension. In case of coil springs, it is a very demanding process. However, with modern suspensions, which utilize properties of hydraulic fluids or high-pressure gases, the demands are lower. Majority of publications concerned with the vehicle suspension are about active suspension [3-5] or fully active suspension (FAS). With FAS, there is a continuous flow of information between all suspension units and ECU along with ABS and all units of the vehicle, which can benefit from the information. Therefore, the breaking distance could be shortened, motions of the vehicle with respect to passengers could be reduced, handling might be improved etc. A small revolution within the vehicle suspension was created by Bose [6]. Though the design overtook all of competition, it featured lots of drawbacks. Overcoming those required new and modern parts. Thus, the production and sales had to wait for the further development of the needed parts. The concept of electromagnetic motors and power amplifiers was given to the prestigious American University and the further development with modern technology and processes is expected. In a meantime, all of the mentioned technology and FAS was successfully implemented in 2018 Audi A8 [7]. Forces generated by the road on the wheels and transferred to the suspension units are for the particular car $3.7 \mathrm{kN}$ in the front and $3.3 \mathrm{kN}$ at the rear side. The electromechanical system of each suspension unit is capable of generating $16 \mathrm{kN}$, with bandwidth ranging from 0 to $6 \mathrm{~Hz}$, meaning that each suspension unit can be adjusted every $15 \mathrm{~ms}$. Another interesting feature is an active roll stabilizer, through the lever $1100 \mathrm{Nm}$ can be exerted to equalize the roll motion of the vehicle. Due to high number of active elements, the ECU required more power for the calculations and control processes. Thus, an extra $48 \mathrm{~V}$ can be found in the vehicle. Presented in Table 1 [2] is the comparison of the chosen parameters of various suspension types. In case of $8 \mathrm{x} 8 \mathrm{APC}$ it is possible to have the FAS system utilized, unfortunately it is not expected that its benefits would overcome the drawbacks (high power demands, complexity of the system, high cost, lower dependability), not without the further development.

Comparison of the most used types of suspensions due to chosen parameters

\begin{tabular}{|c|c|c|c|c|}
\hline Parameters & $\begin{array}{c}\text { Passive } \\
\text { suspensions }\end{array}$ & $\begin{array}{c}\text { Semi-active } \\
\text { suspensions }\end{array}$ & $\begin{array}{c}\text { Hydraulic or } \\
\text { pneumatic active } \\
\text { suspensions }\end{array}$ & $\begin{array}{c}\text { Electromagnetic } \\
\text { active suspensions }\end{array}$ \\
\hline Structure & Simplest & Complex & Most complex & Simple \\
\hline Weight or volume & Lowest & Low & High & Highest \\
\hline Cost & Lowest & Low & Highest & Best \\
\hline Ride comfort & Bad & Medium & Good & Best \\
\hline Handling performance & Bad & Medium & Medium & Good \\
\hline Reliability & Highest & High & Medium & Yes \\
\hline Dynamic performance & Passive & Passive & No & No \\
\hline Energy regeneration & No & No & Yes & \\
\hline
\end{tabular}

\section{Comparison of active and passive suspension}

In case of creating mathematical models of wheeled vehicles, it is recommended to fine-tune them progressively and not to start with full models with $10+$ degrees of freedom (DOF). In the majority of scientific publications, the primal mathematical model is the 2 DOF quarter car model with two possible vertical motions of sprung and unsprung mass. Gradually, the next step is the half-car (4 DOF) model, and full car model with 7 DOF - 1 vertical motion for each unsprung mass and bounce, pitch and roll motions of sprung mass. After successful creating a full car model and trying out various control algorithms, it may be extended to $8 \times 8$ platform with sprung seats with even more DOF. With this procedure, the computing time is slowly building up, so the mistakes are progressively removed. Presented in Fig. 2 is the 2 DOF quarter car model, where $x_{0,1,2}$ are vertical deflections of road, unsprung and sprung masses, $k_{t}$ and $b_{t}$ are tire stiffness and damping coefficient, $k_{a}$ and $b_{a}$ are axle stiffness and damping coefficients. For active suspension, the actuator is illustrated as well. 


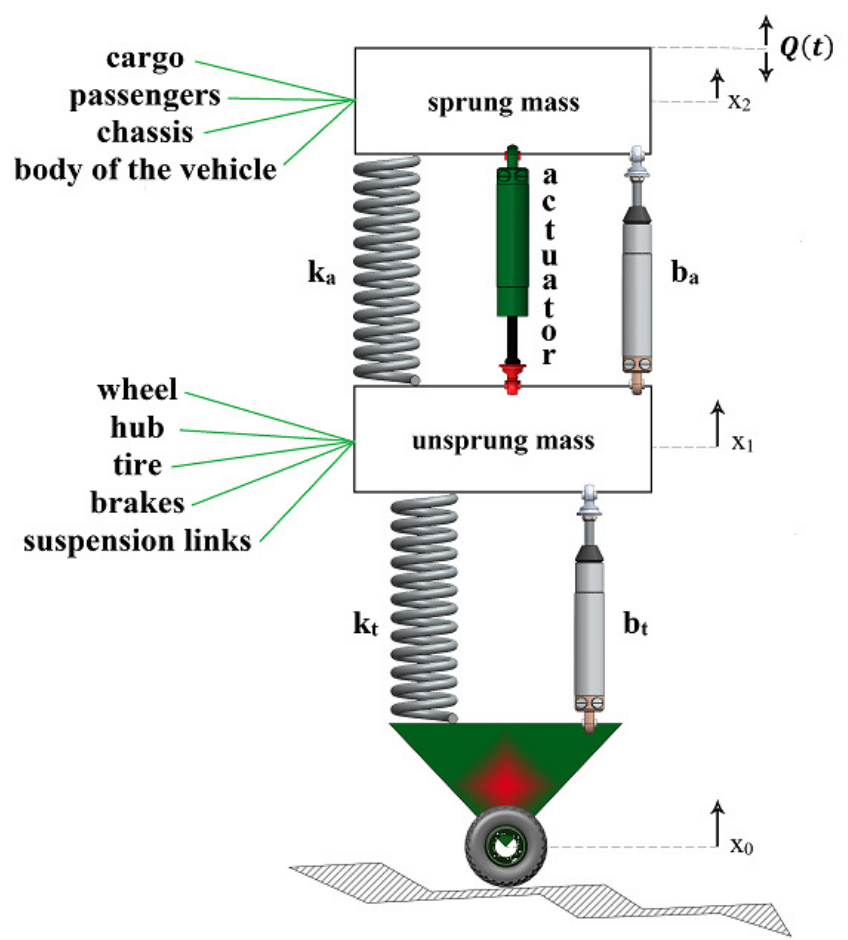

Fig. 2. Quarter car model with 2 DOF

From the basics of applied mechanics, the model might be created by utilization of Lagrange's equations of the motion of second kind (method of accelerating forces). The principle of this method is the law of energy preservation with partial derivations of generalized coordinate $(q)$. Thus, the equation is created:

$$
\frac{d}{d t}\left(\frac{\partial E k}{\partial \dot{q}}\right)-\frac{\partial E k}{\partial q}+\frac{\partial E d}{\partial \dot{q}}+\frac{\partial E p}{\partial q}=Q(t),
$$

where $E k$ - kinetic energy, J;

$E p$ - potential energy, J;

$E d$ - dissipative energy, $\mathrm{J}$;

$q$ - generalized coordinate, $\mathrm{m}$;

$Q(t)$ - generalized force, $\mathrm{N}$.

After the adjustment, linear differential equations of the second order are obtained. Considering the high number of DOF, the matrix notation is preferred:

$$
M \ddot{x}+B \dot{x}+K x=Q(t),
$$

where $M$ - matrix of weight;

$B$ - matrix of damping;

$K$ - matrix of stiffness;

$\ddot{x}, \dot{x}, x-$ column vector of acceleration, velocity and deflection;

$Q(t)$ - vector of external excitation forces.

With utilization of university tools from Quanser and National Instruments- LabVIEW, Fig. 3 was created. The comparison of amplitudes for the active and passive suspension is presented. Vertical red line is marking the change from passive to active suspension. Simulated road input, dashed line, is the same for both configurations; however, the amplitudes of sprung and unsprung masses (top plate and middle plate) have changed significantly. In case of active suspension, sprung mass is almost unaffected by the road input and the amplitude of unsprung mass is lower and damped faster. Semiactive suspension would have amplitudes somewhere in between the mentioned systems. With these tools, various control algorithms can be compared among each other, or with the active suspension. 


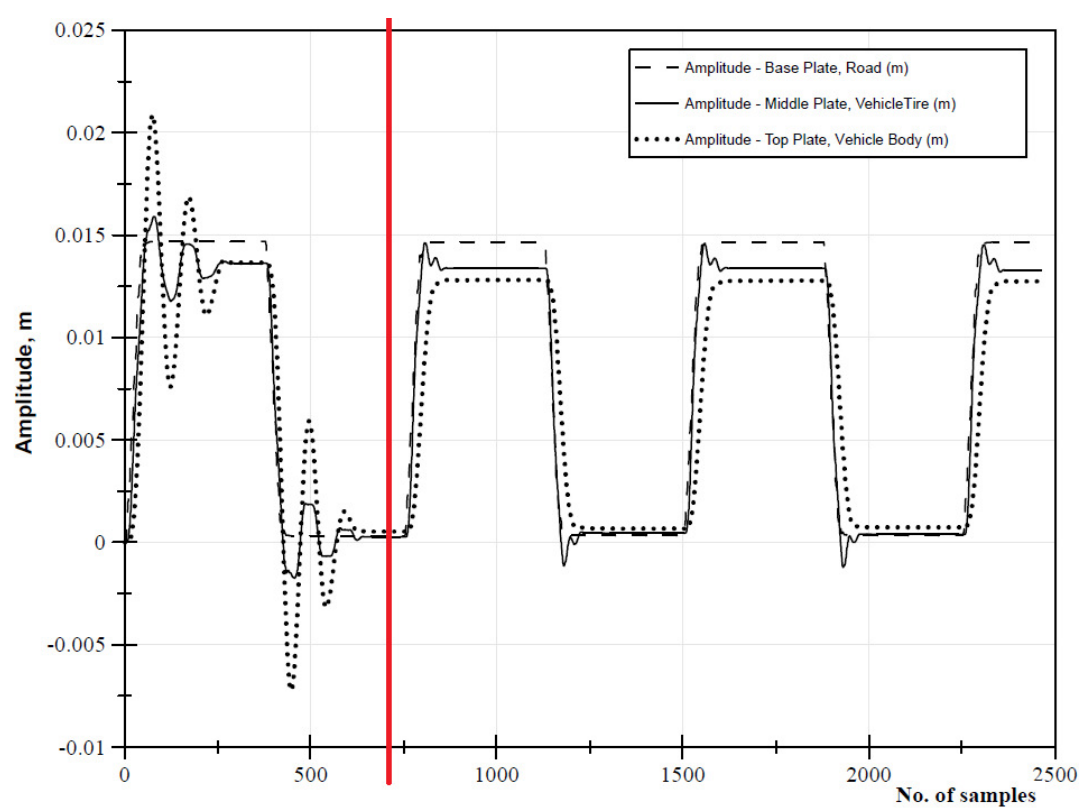

Fig. 3. Comparison between active- left side and passive-right side suspension

\section{Effects of vibrations on a human body}

An issue of effects of vibrations on a human body- performance, attention, physiological health issues, is primarily dealt with in case of truck drivers, construction machinery operators, and many more professional drivers. A general overview can be found in articles [9; 10]. In case of APC, the driver is a very important person, however, the primal role of APC is to safely transport the given numbers of infantry from/on battlefield. If the transport is heading for a battlefield, it is required that infantry be in a good mental a physiological condition, without fatigue, temporary loss of attention and other effects that can be caused by vibrations and can reduce combat readiness of the infantry. A graph of zones with various effects of vibrations on an exposed human body, based on a weighted acceleration and exposure hours is presented in Fig. 4 [11].

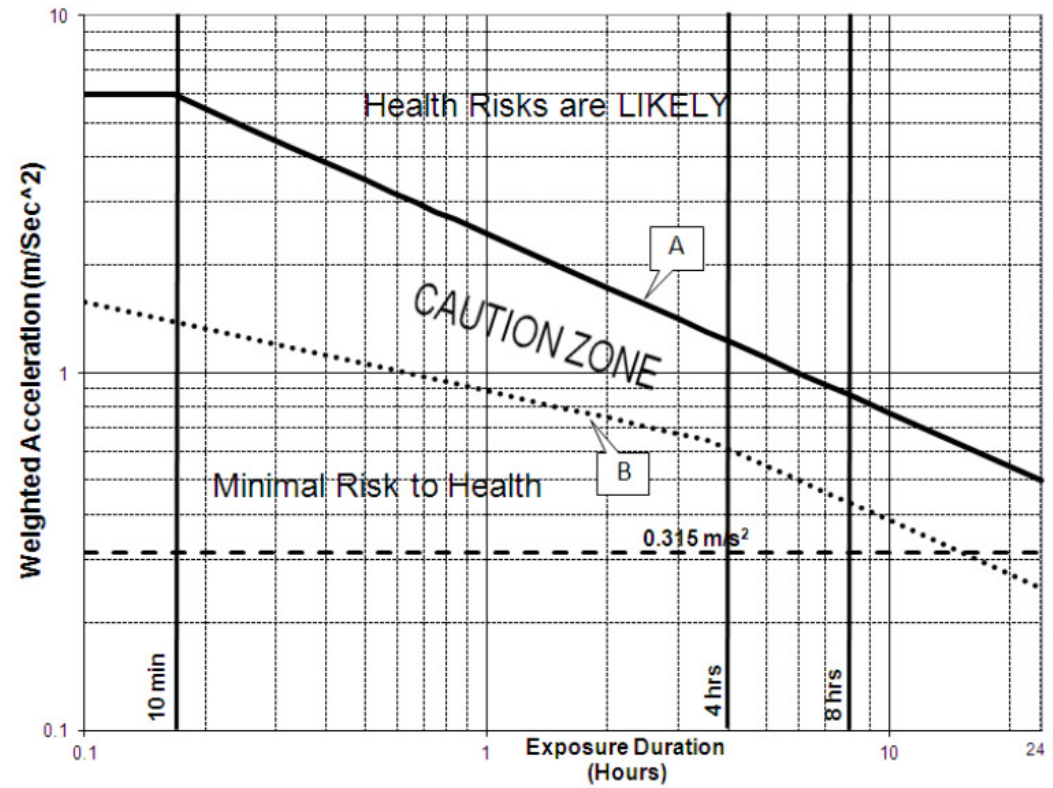

Fig. 4. Zones of possible health risks due to exposure of human body to vibrations

While travelling through the off-road conditions, the crew of the vehicle with passive suspension might be exposed to high doses of vibrations, which might cause the previously stated problems. With respect to dependability of the system and drawbacks of the active suspension, SAS might suit better for APC. 


\section{Conclusions}

Full car mathematical models, even for $6 \times 6$ or $8 \times 8$ configurations, are not breaking news. However, oriented on the high amount of relevant input variables, the number of publications is lower. Moreover, in the case of the Czech Armed Forces, a very interesting vehicle is utilized as APC $-8 \times 8$ PANDUR II. The suspension consists of combined suspension designs, 2 front steered axles with a modified double wishbone suspension and 2 rear axles with a trailing-arm suspension, sprung by torsion bars. Therefore, a full mathematical model will be interesting and will offer a lot of possibilities for SAS control algorithms and even the active suspension might be tried. Multibody software (MBS) by MSC (Adams) will be utilized for verification, along with experimental measures on the vehicle. A proper model of the vehicle is the key to successful implementation of various control algorithms. As the proper model is considered a model with approximately the same measured data for the experiment and mathematical model, where a subtle divergence, adjusted in advance, is allowed.

\section{Acknowledgements}

The presented paper has been prepared with the support of the Ministry of Defence of the Czech Republic, Partial Project for Institutional Development, K-202, Department of Combat and Special Vehicles, University of Defence, Brno.

\section{References}

[1] Savaresi S., Poussot-Vassal Ch., Spelta C., Sename O., Dugard L. Semi-active suspension control design for vehicles. First edition. Oxford: Butterworth-Heinemann, 2010. 240 p.

[2] Tang X., Du H., Sun S., Ning D., Xing Z., Li W. Takagi-Sugeno Fuzzy Control for Semi-Active Vehicle Suspension With a Magnetorheological Damper and Experimental Validation. IEEE/ASME Transactions on Mechatronics [online]. 2017, 22(1), pp. 291-300. DOI: 10.1109/TMECH.2016.2619361.

[3] Coric M., Deur J., Xu L., Tseng H.E., Hrovat D. Optimisation of active suspension control inputs for improved performance of active safety systems. Vehicle System Dynamics [online]. 2017, 56(1), pp. 1-26. DOI: 10.1080/00423114.2017.1340652.

[4] Mohammadi Y., Soheil G. Quarter car active suspension system: Minimum time controller design using singular perturbation method. International Journal of Control, Automation and Systems [online]. 2017, 15(6), pp. 2538-2550. DOI: 10.1007/s12555-016-0608-3.

[5] Kilicaslan S. Control of active suspension system considering nonlinear actuator dynamics. Nonlinear Dynamics [online]. 2018, 91(2), pp. 1383-1394. DOI: 10.1007/s11071-0173951-x.

[6] Jones W.D., "Easy ride: Bose Corp. uses speaker technology to give cars adaptive suspension.”, IEEE Spectrum, vol. 42, no. 5, pp. 12-14.

[7] Adcock I. "Audi details new A8 active suspension", Automotive engineering, Jul. 2017.

[8] Xue X. D., Cheng K. W. E., Zhang Z., Lin J. K., Wang D. H., Bao Y. J., Wong M. K., Cheung N. Study of art of automotive active suspensions. In: 2011 4th International Conference on Power Electronics Systems and Applications [online]. IEEE, 2011, 2011, pp. 1-7. DOI: 10.1109/PESA.2011.5982958.

[9] Sagberg F., Jackson P., Krüger H. P., Muzet A., Williams A. Fatigue, sleepiness and reduced alertness as risk factors in driving. Oslo: Institute of Transport Economics, 2004.

[10] Troxel W. M., Helmus T. C., Tsang F., Price C. C. "Evaluating the Impact of Whole-Body Vibration (WBV) on Fatigue and the Implications for Driver Safety", 2015.

[11] MIL-STD-1472G: Department of Defense Design Criteria Standard: Human Engineering. 2012. 